\title{
Long-Term Follow-up Results of Fluorescence and Blue Dye Guided Sentinel Lymph Node Biopsy in Early Breast Cancer
}

\section{Chaobin Wang}

Peking University People's Hospital

\section{Fuzhong Tong}

Peking University People's Hospital

\section{Yingming Cao}

Peking University People's Hospital

\section{Peng Liu}

Peking University People's Hospital

\section{Bo Zhou}

Peking University People's Hospital

\section{Hongjun Liu}

Peking University People's Hospital

\section{Lin Cheng}

Peking University People's Hospital

\section{Miao Liu}

Peking University People's Hospital

\section{Jiajia Guo}

Peking University People's Hospital

\section{Fei Xie}

Peking University People's Hospital

Houpu Yang

Peking University People's Hospital

\section{Siyuan Wang}

Peking University People's Hospital

Shu Wang ( $\nabla$ shuwang@pkuph.edu.cn )

Peking University People's Hospital https://orcid.org/0000-0002-8651-4795

\section{Yuan Peng}

Peking University People's Hospital 
Keywords: Breast cancer, Sentinel lymph node biopsy, Indocyanine green, Axillary recurrence, Lymphedema

Posted Date: February 17th, 2021

DOl: https://doi.org/10.21203/rs.3.rs-229024/v1

License: (c) (1) This work is licensed under a Creative Commons Attribution 4.0 International License. Read Full License

Version of Record: A version of this preprint was published at Breast Cancer Research and Treatment on March 24th, 2021. See the published version at https://doi.org/10.1007/s10549-021-06196-6. 


\section{Abstract}

Purpose This study aimed to assess the value of the combination of indocyanine green (ICG) and methylene blue (MB) dye in early breast cancer patients undergoing sentinel lymph node biopsy (SLNB).

Methods Between January 2011 and December 2015, 1061 early breast cancer patients underwent SLNB were included. SLNB was performed for enrolled patients by injection of both ICG and MB. Detection rate, positivity, and number of sentinel lymph nodes (SLNs) by ICG and MB were analysed. Axillary recurrence and arm lymphedema at 5.6 years follow-up were reported.

Results The combination of ICG and MB resulted in a very high detection rate of $99.6 \%$, the median number of sentinel lymph nodes was 3 . A total of 374 histologically confirmed positive SLNs were detected in 237 patients, $96.6 \%$ of the positive patients and $94.1 \%$ of the positive nodes could be identified by the combination of ICG and MB. After a median follow-up of 5.6 (2 to 9.3 ) years, $0.64 \%$ of patients with negative SLNs had ipsilateral axillary recurrence, and the incidence of arm lymphedema was $2.1 \%$.

Conclusions Although the missing isotope control weakens the interpretation of the findings, the dual tracing modality of ICG and MB, without involvement of radioactive isotopes, was an effective method for SLNB in patients with early breast cancer.

\section{Introduction}

Sentinel lymph node biopsy (SLNB) was first applied in breast cancer care in the 1990s by Krag et al [1], and now has become the standard procedure for patients with clinically negative axilla [2]. SLNB can accurately stage axillary lymph node metastasis and avoid unnecessary axillary lymph node dissection (ALND) [3], and the risk of lymphedema, paresthesia, and pain syndromes is reduced subsequently [4].

The existing tracer-based techniques for SLNB have involved blue dye, technetium-99m (99mTc)-labeled nanocolloid, or both [5]. Isosulfan blue and patent blue have been the most commonly used blue dyes in developed countries and methylene blue has been widely used in developing countries like China [6, 7]. A dual tracer of blue dye and radioisotope (RI) can improve detection of SLNs and reduce the false negative rate (FNR) [8] and thus has been recommended as the standard technique in guidelines [9]. However, the use of RI creates some logistical challenges for hospitals, such as the handling and disposal of isotopes, training of staff, legislative requirements, and the reluctance of patients to be exposed to radiation [9]. The application of RI is limited to breast centers with RI facilities and nuclear medicine [10]. And the timewindow for SLN identification is limited due to the short half-life (6 hours) of 99mTechnetium [9]. Although SLNB was performed routinely in $76 \%$ of hospitals in China, only $14.6 \%$ of hospitals used dualtracer method including RI [11]. It may be due to the inconvenience of RI application. Constraints of radioisotopes have led to the development of non-radioactive alternatives. Innovative tracers such as indocyanine green (ICG), superparamagnetic iron oxide (SPIO), and microbubbles have been explored [12]. The main disadvantages of SPIO include the need to remove metal retractor from the wound during 
surgery to obtain magnetometer readings, and SPIO may interfere with contrast-enhanced MRI [13]. It was reported that the standard dual technique of $\mathrm{RI}$ and blue dye was significantly better than microbubbles in terms of SLNs identification rate [9]. ICG was one of the most promising technique because of the high SLNs identification rate (93\%-100\%) and was the most commonly used non-radioactive tracer in clinical trials of SLNB [12]. ICG is a FDA-approved organic molecule with near infrared (NIR) fluorescence [14]. SLNB with NIR fluorescence imaging using ICG was first reported by Kitai et al, and showed high detection rate for SLN with the rate of $94 \%$ [15]. As ICG can be transcutaneously detected similar to radioactive agents, the combination of ICG and methylene blue (MB) shows great potential as an alternative to standard dual mapping methods involving RI. Previously, we reported that dual tracer of MB and ICG improved the mapping performance in 198 patients with early breast cancer [16]. Now we report the final results for 1061 patients from this prospective cohort study, not only for SLN detection, but also for long-term axillary recurrence.

\section{Methods}

\section{Study Design and Patients}

The study was registered retrospectively as Australia and New Zealand Clinical Trials Registry No.12612000109808 in January 2012. The first participant was enrolled in January 2011. From January 2011 to May 2012, we conducted a randomized controlled study comparing MB and MB combined with ICG in SLNB for early breast cancer patients according to the registered content. The preliminary results have showed that MB combined with ICG is better than MB alone in identification of SLNs, which has been published in a local journal [17]. Therefore, dual tracers were used for all patients since May 2012. A total of 1061 consecutive early breast cancer patients underwent SLNB with dual tracing by MB and ICG were enrolled in the present study between January 2011 and December 2015. Primary breast cancer was confirmed in eligible patients by core needle biopsy or surgical biopsy, and eligible patients had negative axillary lymph nodes by clinical examination and ultrasound. Patients were not admitted to the study if any of following criteria were present: distant metastasis, inflammatory breast cancer, clinically or radiologically suspicious lymph nodes, previous axillary surgery, and hypersensitivity to iodine or ICG. Patients with previous breast surgery were not excluded. Written informed consents were obtained from all included patients. The study was approved by the Ethics Committee of Peking University People's Hospital, Beijing.

\section{Procedure}

MB (Jizhou Pharmaceutical, Suzhou, China) was diluted in saline to a final concentration of $1 \%$. ICG (Dandong Pharmaceutical, Jilin, China) was dissolved and diluted in distilled water to a final concentration of $0.5 \mathrm{mg} / \mathrm{mL}$ before use, and $1 \mathrm{~mL} \mathrm{MB}$ and $1 \mathrm{~mL}$ ICG were injected intradermally in the periareolar region 5 minutes before surgery. ICG fluorescence was stimulated and detected by the fluorescence detector (MingDe Medicine, China) and lymphatic drainage could be navigated in real time. Blue-stained nodes and fluorescent nodes were removed and deemed SLNs. Nodes that were hard and 
highly suspicious for metastatic tumor were also removed and defined as SLNs irrespective of fluorescence or MB staining. Lymph nodes labeled by MB and/or ICG are shown in Fig. 1. SLNs were classified into four groups by different tracers: ICG(+)/MB(+), ICG(+)/MB(-), ICG(-)/MB(+), and ICG(-)/MB(-). Patients with tumor-positive SLNs had ALND unless they met entry criteria for the ACOSOG Z0011 study. All patients received standard adjuvant therapy including radiotherapy, chemotherapy, and endocrine therapy, according the National Comprehensive Cancer Network (NCCN) guideline.

\section{Outcomes and statistical analysis}

The total numbers of SLNs and the numbers of positive SLNs detected by MB alone and by the combination of MB and ICG were recorded. Patients were followed up every six months. Follow-up was ended on April 15, 2020.

Relapse free survival (RFS) was defined as the time from diagnosis to the recurrence of tumor, including locoregional disease or distant metastasis, or death from any cause. Breast cancer specific survival (BCSS) was defined as the time from diagnosis to death from breast cancer.

Lymphedema was recorded as self-reported lymphedema [18]. Patients were asked to indicate the presence of swelling during follow-up by answering following yes or no questions: 1) Have you experienced swelling of the upper-limb on the breast cancer treated side in the past year?; 2) Is the arm on the treated side thicker than the other arm?; 3) Have you seen a doctor for arm swelling?

The associations between different tracers and identification of SLNs were evaluated using $\chi 2$ tests. Mann-Whitney $U$ tests were used to compare the median of SLNs in different tracers. The Kaplan-Meier method was used to draw survival curves. A 2-sided $p$ value $<0.05$ was considered significant. Statistical analyses were performed with statistical software (SPSS, version 20.0; RStudio, version1.3.1056).

\section{Results}

\section{Patient characteristics}

The present study enrolled 1061 patients with early breast cancer (Fig. 2). Their characteristics are presented in Table 1. 
Table 1

Patient and tumor characteristics

\section{Characteristics}

Total

Age(years)

$53(20-89) *$

$\geq 50$

$<50$

$\mathrm{BMI}$

$\geq 24$

$<24$

Histological type

In situ

Invasive ductal

Specified carcinoma

$\mathrm{HR}$

Positive

Negative

Unknown

HER2

Positive

Negative

Unknown

Molecular subtype (immunohistochemical)

Luminal A

Luminal B
$\mathbf{N}$ 1061
$\%$

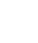




\begin{tabular}{|c|c|c|}
\hline Characteristics & $\mathbf{N}$ & $\%$ \\
\hline HER2 enriched & 90 & $8.5 \%$ \\
\hline Triple negative & 142 & $13.4 \%$ \\
\hline Unknown & 21 & $2.0 \%$ \\
\hline \multicolumn{3}{|l|}{ pT } \\
\hline pTis & 87 & $8.2 \%$ \\
\hline pT1 & 766 & $72.2 \%$ \\
\hline pT2 & 187 & $17.6 \%$ \\
\hline рT3 & 10 & $0.9 \%$ \\
\hline pTX & 11 & $1.0 \%$ \\
\hline \multicolumn{3}{|l|}{ pN } \\
\hline pNO & 803 & $75.7 \%$ \\
\hline pN1 & 214 & $20.2 \%$ \\
\hline pN2 & 34 & $3.2 \%$ \\
\hline pN3 & 10 & $0.9 \%$ \\
\hline \multicolumn{3}{|l|}{ pStage } \\
\hline p0 & 87 & $8.2 \%$ \\
\hline pl & 594 & $56.0 \%$ \\
\hline pll & 324 & $30.5 \%$ \\
\hline plll & 46 & $4.3 \%$ \\
\hline Unknown" & 10 & $0.9 \%$ \\
\hline \multicolumn{3}{|l|}{ Excisional biopsy } \\
\hline Yes & 796 & $75 \%$ \\
\hline No & 265 & $25 \%$ \\
\hline \multicolumn{3}{|l|}{ Surgery } \\
\hline \multicolumn{3}{|c|}{$\begin{array}{l}\text { BMI, Body mass index; HR, Hormone receptor; HER2, Human epidermal growth factor receptor 2; } p \text {, } \\
\text { Pathologic. }\end{array}$} \\
\hline \multicolumn{3}{|c|}{ * Age is the median age, including the maximum and minimum. } \\
\hline $\begin{array}{l}\text { \# Eleven patient } \\
\text { patient with pN2 }\end{array}$ & $\begin{array}{l}\text { then } \\
10 p\end{array}$ & $\begin{array}{l}\text { wn. One } \\
\text { Inknown. }\end{array}$ \\
\hline
\end{tabular}




\begin{tabular}{|c|c|c|}
\hline Characteristics & $\mathbf{N}$ & $\%$ \\
\hline Mastectomy & 600 & $56.6 \%$ \\
\hline Breast conserving & 461 & $43.4 \%$ \\
\hline \multicolumn{3}{|c|}{ Adjuvant chemotherapy } \\
\hline Yes & 665 & $62.7 \%$ \\
\hline No & 396 & $37.3 \%$ \\
\hline \multicolumn{3}{|c|}{ Adjuvant radiotherapy } \\
\hline Yes & 617 & $58.2 \%$ \\
\hline No & 444 & $41.8 \%$ \\
\hline \multicolumn{3}{|c|}{$\begin{array}{l}\text { BMI, Body mass index; HR, Hormone receptor; HER2, Human epidermal growth factor receptor 2; } p \text {, } \\
\text { Pathologic. }\end{array}$} \\
\hline \multicolumn{3}{|c|}{ * Age is the median age, including the maximum and minimum. } \\
\hline \multicolumn{3}{|c|}{$\begin{array}{l}\text { \# Eleven patients received excisional biopsy in other hospital, and pT of them were unknown. One } \\
\text { patient with pN2 was classified as stage plll, and stage of the remaining } 10 \text { patients was unknown }\end{array}$} \\
\hline
\end{tabular}

All of 1061 patients received SLNB traced by combination of ICG and MB. The staining for each lymph node of 92 patients was not recorded clearly by different tracers as ICG(+)/MB(+), ICG(+)/MB(-), ICG(-)/MB(+), or ICG(-)/MB(-). Therefore, the remaining 969 patients were included in the analysis for sentinel lymph node detection.

SLNs were identified in 910 (93.9\%) patients by MB alone, and in 965 (99.6\%) patients by the combination of ICG and MB $(p<0.001)$ (Table 2$)$. The dual tracer had a significantly higher sentinel node detection rate than $\mathrm{MB}$ alone, and the absolute difference was $5.7 \%$. Older age, higher $\mathrm{BMI}$, upper outer quadrant tumor, prior excisional biopsy, and larger tumor size were associated with a reduced detection rate with MB alone but not with the combination method (Online Resource 1).The combination method also resulted in the identification of more lymph nodes in each patient (Table 2). The median number of SLNs detected by MB alone was 2, while the median number of SLNs detected by the dual tracer was 3 $(p<0.001)$. 
Table 2

Comparison of identification by different tracers

\begin{tabular}{|llll|}
\hline & MB & ICG + MB & P \\
\hline Identification rate & $910 / 969(93.9 \%)$ & $965 / 969(99.6 \%)$ & $<0.01$ \\
\hline Positive SLNs identified & $259 / 374(69.3 \%)$ & $352 / 374(94.1 \%)$ & $<0.01$ \\
\hline Patients with N (+) identified & $201 / 237(84.8 \%)$ & $229 / 237(96.6 \%)$ & $<0.01$ \\
\hline Patients with more than 2 N (+) & $9(0.9 \%)$ & $28(2.9 \%)$ & $<0.01$ \\
\hline SLNs, Sentinel lymph nodes; MB, Methylene blue; ICG, Indocyanine green. & \\
\hline
\end{tabular}

A total of 374 histologically confirmed positive SLNs were detected in 237 patients, of which $84.8 \%$ (201/237) could be identified by MB, and $96.6 \%$ (229/237) by dual tracers, resulting in an additional 28 $(11.8 \%)$ node positive patients identified by the dual tracer $(p<0.001)$ (Table 2$)$. Among the 374 positive nodes excised, $69.3 \%$ (259/374) could be identified by MB alone and $94.1 \%(352 / 374)$ by the dual tracer $(p<0.001)$. Among 28 patients who had more than 2 positive SLNs detected by dual tracer, $19(67.9 \%)$ would have been identified as $\leq 2$ if using MB alone.

\section{Survival}

Among 1061 patients, 32 were lost to follow-up; therefore, 1029 patients were included in the survival analysis. After a median follow-up of 5.6 years (2 to 9.3 years), there were 73 RFS events including 38 recurrences without death, 19 deaths with recurrence, and 16 deaths without recurrence. The 6-year RFS was $93.5 \%$ in node negative patients and $91.3 \%$ in node positive patients $(p=0.21)$ and the 6 -year BCSS was $97.8 \%$ and $97.7 \%(p=0.47)$ (Fig. 3$)$.

Five of 777 patients with negative SLNs were diagnosed with ipsilateral axillary recurrences as the first events after a median follow-up of 5.6 years. The ipsilateral axillary recurrence rate was $0.64 \%$ (Online Resource 2).

\section{Lymphedema}

Details on lymphedema were collected from 973 of 1061 patients. Among 766 patients who had SLNB, 16 reported ipsilateral arm lymphedema and among 207 patients with ALND, 29 reported lymphedema. The incidence of upper extremity lymphedema following SLNB was $2.1 \%$ and was significantly lower versus ALND $(14 \%)(p<0.001)$.

\section{Discussion}

The present prospective study reports a radioisotope-free method of SLNB using a combination of ICG and MB that had a $99.6 \%$ detection rate. After a median follow-up of 5.6 years, the ipsilateral axillary recurrence rate in patients with negative SLNs was $0.64 \%$ and the incidence of upper extremity arm 
lymphedema was $2.1 \%$. The dual tracing modality of ICG and MB, without radioactive isotopes, was an effective method for SLNB in patients with early breast cancer.

Although blue dye has no radiation exposure and is not expensive, its injections may cause skin necrosis and anaphylaxis [19], which was absent when ICG was used. The approach of blue dye was cheap but achieved lower identification rate. Both RI and ICG could increase the detection rate but need additional apparatus which means increased cost [8,9]. Previous studies have compared the cost of RI and ICG, which showed \$331 - \$420 per patient for RI and \$5 - \$111 per patient for ICG [10], and it cost \$18 per patient in China similarly. It seemed a little bit cheaper for ICG than RI, however, we could not conclude ICG is more cost-effective than RI. Based on our practical experience, the use of ICG made the surgical operation more visualized, it's easier and quicker for us to find the SLN. But we did not have accurate records of the surgery time. Other study have reported that the average time between skin incision and SLN identification was $7 \pm 6$ min when using ICG [20]. And it has been showed in a randomized controlled trial that the time to detect the first SLN was similar between multimodal method (combination of RI, ICG and blue dye) and RI alone (6.5 \pm 5.16 vs. $8.0 \pm 4.35 \mathrm{~min} ; \mathrm{p}=0.13)$ [21].

The fluorescent ICG tracer helps to visualize lymphatic drainage from breast to axilla for incision localization and SLN identification, and, as in our study, previous trials have shown detection rates as high as 99 percent $[22,23]$. Although age, BMI, tumor location, and excisional biopsy have all been reported to associate with decreased SLN detection rates [24-27], we achieved very high sensitivity in our study independent of negative factors when ICG and MB were used together.

FNR is a crucial indicator for assessing the accuracy of SLNB. However, FNR was not analyzed in our study. Since MB was already considered a reliable tracer method in China [7], and no additional ALND was performed in patients with negative SLNs, calculating the FNR of ICG and MB would have been difficult.

Instead, the efficacy of SLNB traced by combination of ICG and MB was evaluated in terms of long term ipsilateral axillary recurrence. Although previous studies have examined the rate of ipsilateral axillary recurrence after SLNB with ICG tracer, they have enrolled relatively few patients and the time of follow-up was short $[28,29]$. In our study, after a median follow-up of 5.6 years, the ipsilateral axillary recurrence rate of was $0.64 \%$, which was comparable to the result reported in the NSABP B32 trial and previous studies using different tracers (Table 3) [28-32]. 
Table 3

Ipsilateral axillary recurrence rates

\begin{tabular}{|c|c|c|c|c|}
\hline Author & Tracers & $\begin{array}{l}\text { Patients with SLNB } \\
\text { alone }\end{array}$ & $\begin{array}{l}\text { Follow-up } \\
\text { time }\end{array}$ & $\begin{array}{l}\text { Axillary recurrence } \\
\text { rate }\end{array}$ \\
\hline Krag et al ${ }^{[30]}$ & RI + blue dye & 2011 & $8 y$ & $0.4 \%$ \\
\hline Inoue et al ${ }^{[28]}$ & $\begin{array}{l}\text { ICG + blue } \\
\text { dye }\end{array}$ & 464 & $3.2 y$ & $0.4 \%$ \\
\hline Boniface et al[ ${ }^{[31]}$ & RI + blue dye & 2216 & $10.5 y$ & $1.6 \%$ \\
\hline $\begin{array}{l}\text { Galimberti et } \\
\text { al }^{[32]}\end{array}$ & $\mathrm{RI}$ & 5262 & $7.0 y$ & $1.7 \%$ \\
\hline Toh et al ${ }^{[29]}$ & ICG & 153 & $2.5 y$ & 0 \\
\hline Our study & $\begin{array}{l}\text { ICG + blue } \\
\text { dye }\end{array}$ & 777 & $5.6 y$ & $0.67 \%$ \\
\hline
\end{tabular}

The number of SLNs that should be excised for accurate lymph node staging is controversial. Is detection of relatively more SLNs helpful in accurate axillary staging? And how many SLNs are enough? Several studies suggested that SLNB should not remove only one or two lymph node(s) when other labeled nodes are present, and in cases where 4 or more SLNs were removed accuracy reached $100 \%$ and the FNR dropped to zero $[33,34]$. In patients undergoing SLNB after neoadjuvant chemotherapy, a dual tracer reached an acceptable FNR when more than 2 SLNs were detected [35]. In our study, the median number of SLNs detected by the combined method was 3 , a little bit higher than traditional methods. It was reported that more SLNs identified by ICG was probably due to the low molecular weight and high degree of diffusion of ICG allowing it to spread beyond the SLN to secondary draining lymph node [36]. ICG could bind rapidly and completely to proteins. After intradermal or subcutaneous injection, ICG could theoretically bind to proteins and consequently became larger molecular tracer [37]. And it has been shown in a double-blind randomized trial that the average number of detected SLNs by ICG or ICG:HSA (adsorption of ICG to human serum albumin) were not significantly different [37]. We think the more reasonable explanation for a little bit more SLNs was due to the higher sensitivity of ICG method. It does not rely on visual search for dye but on light of fluorescence. Fluorescence can penetrate through thin layers of tissue and reveal nodes otherwise hidden when using MB [14]. We believe that the dual tracer with ICG and MB would be useful in SLNB, even after neoadjuvant chemotherapy. Further studies will be needed.

Our study showed that the detection rate of positive lymph nodes was $69.3 \%$ by MB alone. More positive lymph nodes could be detected by dual tracers. However, it was comparable with the results of NSABP B32 study. The detection rate of positive lymph nodes was $77.5 \%$ and $93 \%$ by blue dye and combined method, respectively [24]. The number of positive lymph nodes at SLNB is very important for subsequent 
decision making. Patients undergoing breast-conserving surgery with only 1 or 2 positive SLNs who meet the entry criteria for the ACOSOG Z0011 trial can be exempted from ALND [38]. In our study, among 28 patients with more than 2 positive SLNs detected by the dual tracer, $67.9 \%$ had only 2 or less positive SLNs detected by MB alone. Therefore, the application of ICG could be helpful in accurate decision making with a higher number of positive SLNs.

Some may argue that excision of more SLNs detected by ICG will increase the incidence of lymphedema. The present study is the first one to report the incidence of lymphedema after SLNB with ICG tracer in a large sample cohort. The incidence of breast cancer treatment-related lymphedema following SLNB was $2.1 \%$, whereas that following ALND was $14 \%$. Goldberg reported on a series of 600 patients received SLNB by combined dye-isotope mapping technique, the incidence of patient-reported lymphedema was $3 \%$ [39]. And another report showed that the incidence of subjective lymphedema after SLNB was $4.1 \%$ [40]. Therefore, from the results of our study, it seems that SLNB with a dual tracer of combined ICG and $\mathrm{MB}$ is not related to an increased risk of lymphedema.

Our study has some shortcomings. RI has not been available in the majority of the hospitals in China, and it is regrettable we did not compare the dual ICG and MB tracer with the standard method of RI and blue dye recommended by the guidelines. We have also noticed that several studies compared the combination of ICG and RI to RI and blue dye [10]. with the purpose of replacing blue dye with ICG in the dual tracer method. Clearly, this was not our goal. Since previous studies have shown that ICG performed similarly to RI with regard to the number of SLNs identified, rate of failed mapping, and identification of pathologically positive SLNs [41, 42], the hypothesis of replacing RI by ICG in a dual tracer method was reasonable.

\section{Conclusion}

Although the missing isotope control weakens the interpretation of the findings, the dual tracing modality of ICG and MB, without involvement of radioactive isotopes, was an effective method in SLNB for patients with early breast cancer.

\section{Declarations}

Authors' contributions: Chaobin Wang participated in the study design and coordination and participated in the data analysis. Shu Wang conceived the study and participated in its design and coordination. Fuzhong Tong, Yingming Cao, Peng Liu, Bo Zhou, Hongjun Liu, Lin Cheng, Miao Liu, Jiajia Guo, Fei Xie, Houpu Yang, Siyuan Wang, Yuan Peng participated in the clinical study implementation and data collection. All authors approved the final manuscript.

Fundings: This study was sponsored by Capital's Funds for Health Improvement and Research (2014-24083), Capital's Funds for Health Improvement and Research (2009-3034), Natural Science Foundation of Beijing Municipality (2150000028). 
Conflict of interest: The authors declare that they have no conflict of interest.

Ethics approval: This study was approved by the Ethical Committee of Peking University People's Hospital, Beijing.

Consent to participate: Written informed consent was obtained from all patients.

\section{References}

1. . Krag DN, Weaver DL, Alex JC et al (1993) Surgical resection and radiolocalization of the sentinel lymph node in breast cancer using a gamma probe. Surg Oncol 2(6):335-339

2. . Manca G, Rubello D, Tardelli E et al (2016) Sentinel Lymph Node Biopsy in. Breast Cancer Indications, Contraindications, and. Controversies. Clin Nucl Med 41:126-133

3. . Lyman GH, Sarah T, Edge SB et al (2014) Sentinel lymph node biopsy for patients with early-stage breast cancer: American Society of Clinical Oncology clinical practice guideline update. J Clin Oncol 32:1365-1383

4. . Disipio T, Rye S, Newman B et al (2013) Incidence of unilateral arm lymphoedema after breast cancer: a systematic review and meta-analysis. Lancet Oncol 14:500-515

5. . Giammarile, Francesco E et al (2013) The EANM and SNMMI practice guideline for lymphoscintigraphy and;sentinel node localization in breast cancer. Eur J Nucl Med Mol Imaging 40:1932-1947

6. . Simmons R, Thevarajah S, Meghan B. Brennan et al (2003) Methylene Blue Dye as an Alternative to Isosulfan Blue Dye for Sentinel Lymph Node Localization. Ann Surg Oncol 10(3):242-247

7. . Li J, Chen X, Qi M et al (2018) Sentinel lymph node biopsy mapped with methylene blue dye alone in patients with breast cancer: A systematic review and meta- analysis. PLoS One 13(9):e0204364

8. . David BY, Syme JP, Collins G, Bruce Mann et al (2005) Comparison of blue dye and isotope with blue dye alone in breast sentinel node biopsy. ANZ J Surg 75:817-821

9. . Muneer A, Purushotham AD, Michael D (2014) Novel techniques for sentinel lymph node biopsy in breast cancer: a systematic review. Lancet Oncol 15:e351-e362

10. Goonawardena J, Yong C, Law M (2020) Use of indocyanine green fluorescence compared to radioisotope for sentinel lymph node biopsy in early-stage breast cancer: systematic review and meta-analysis. Am J Surg S0002-9610(20):30069-30066

11. Benlong Yang G, Ren E, Song et al (2020) Current Status and Factors Influencing Surgical Options for Breast Cancer in China: A Nationwide Cross-Sectional Survey of 110 Hospitals. Oncologist 25:e1473-e1480

12. Goyal A (2018) New Technologies for Sentinel Lymph Node Detection. Breast care 13(5):349-353

13. Eleftherios P, Mamounas T, Kuehn, Emiel JT, Rutgers et al (2017) Current approach of the axilla in patients with early-stage breast cancer. Lancet 14;S0140-6736(17):31451-31454 
14. . Cousins a A, Sarah K, Thompson A, Bruce Weddin et al (2014) Clinical relevance of novel imaging technologies for sentinel lymph node identification and staging. Biotechnol Adv 32(2):269-279

15. Toshiyuki Kitai T, Inomoto M, Miwa et al (2005) Fluorescence navigation with indocyanine green for detecting sentinel lymph nodes in breast cancer. Breast Cancer 12(3):211-215

16. Guo J, Yang H, Shu W et al (2017) Comparison of sentinel lymph node biopsy guided by indocyanine green, blue dye, and their combination in breast cancer patients: a prospective cohort study. World $\mathrm{J}$ Surg Oncol 15(1):196

17. Cao yingming, Wang shu, Guo jiajia et al (2014) Combination of ICG and methylene blue for mapping sentinel lymph nodes in early breast cancer patients. Chinese Journal of General Surgery 29(2):119-122

18. Lopez Penha TR, Slangen JJG, Heuts EM et al (2011) Prevalence of lymphoedema more than five years after breast cancer treatment. Eur J Surg Oncol 37(12):1059-1063

19. Efron P, Knudsen E, Hirshorn S et al (2002) Anaphylactic reaction to isosulfan blue used for sentinel node biopsy: case report and literature review. Breast J 8:396e399

20. Verbeek FP, Troyan SL, Mieog JS et al (2014) Near-infrared fluorescence sentinel lymph node mapping in breast cancer: a multi- center experience. Breast Cancer Res Treat 143:333e342

21. Jung S-Y, Kim S-K, Kim SW et al (2014) Comparison of Sentinel Lymph Node Biopsy Guided by the Multimodal Method of Indocyanine Green Fluorescence, Radioisotope, and Blue Dye Versus the Radioisotope Method in Breast Cancer: A Randomized Controlled Trial. Ann Surg Oncol 21:12541259

22. Tomoharu Sugie T, Sawada N, Tagaya et al (2013) Comparison of the Indocyanine. Green Fluorescence and Blue Dye Methods in Detection of Sentinel Lymph Nodes in Early-stage Breast Cancer. Ann Surg Oncol 20:2213-2218

23. Akira Hirano M, Kamimura K, Ogura et al (2012) A Comparison of Indocyanine Green. Fluorescence Imaging Plus Blue Dye and Blue Dye Alone for Sentinel Node Navigation Surgery in Breast Cancer Patients. Ann Surg Oncol 19:4112-4116

24. David N, Krag SJ, Anderson, Thomas B, Julian et al (2007) Technical outcomes of sentinel-lymphnode resection and conventional axillary-lymph-node dissection in patients with clinically nodenegative breast cancer: results from the NSABP B-32 randomised phase III trial. Lancet Oncol 8:881888

25. Lorraine Tafra DR, Lannin, Melvin S, Swanson et al (2001) Multicenter Trial of Sentinel Node Biopsy for Breast Cancer Using Both Technetium Sulfur Colloid and Isosulfan Blue Dye. Ann surg 233(1):51-59

26. Anees B, Chagpar RC, Martin CR, Scoggins et al (2005) Factors predicting failure to identify a sentinel lymph node in breast cancer. Surgery 138:56-63

27. Cox CE, Dupont E, George F, Whitehead et al (2002) Age and Body Mass Index May Increase the Chance of Failure in Sentinel Lymph Node Biopsy for Women with Breast Cancer. Breast J 8(2):8891 
28. Tomoo Inoue T, Nishi Y, Nakano et al (2016) Axillary lymph node recurrence after. sentinel lymph node biopsy performed using a combination of indocyanine green fluorescence and the blue dye method in early breast cancer. Breast cancer 23(2):295-300

29. U.Toh N.Iwakuma MMishima et al (2015) Navigation surgery for intraoperative sentinel lymph node detection using Indocyanine green (ICG) fluorescence real-time imaging in breast cancer. Breast Cancer Res Treat 153:337-344

30. David N, Krag SJ, Anderson, Thomas B, Julian et al (2010) Sentinel-lymph-node resection. compared with conventional axillary-lymph-node dissection in clinically node-negative patients with breast cancer: overall survival findings from the NSABP B-32 randomised phase 3 trial. Lancet Oncol 11:927-933

31. Boniface F, Bergkvist et al (2017) Ten-year report on axillary recurrence after negative sentinel node biopsy for breast cancer from the Swedish Multicentre Cohort Study. Br J Surg 104(3):238-247

32. Galimberti M, Maisonneuve et al (2014) Long-term follow-up of 5262 breast cancer patients with negative sentinel node and no axillary dissection confirms low rate of axillary disease. Eur J Surg Oncol 40(10):1203-1208

33. Yi M, Meric-Bernstam F, Merrick I. Ross et al (2008) How Many Sentinel Lymph Nodes Are Enough During Sentinel Lymph Node Dissection for. Breast Cancer? Cancer 113(1):30-37

34. Ban EJ, Lee JS, Koo JS et al (2011) How Many Sentinel Lymph Nodes Are Enough for Accurate Axillary Staging in T1-2 Breast Cancer? J Breast Cancer 14(4):296-300

35. Kuehn B, Fehm et al (2013) Sentinel-lymph-node biopsy in patients with breast cancer before and after neoadjuvant chemotherapy (SENTINA): a prospective, multicentre cohort study. Lancet Oncol 14:609-618

36. Hojo T, Nagao T, Kikuyama M et al (2010) Evaluation of sentinel node biopsy by combined. fluorescent and dye method and lymph flow for breast cancer. Breast 19:210-213

37. Merlijn Hutteman J, Sven D, Mieog, Joost R, van der Vorst et al (2011) Randomized, double-blind comparison of indocyanine green with or without albumin premixing for near-infrared fluorescence imaging of sentinel lymph nodes in breast cancer patients. Breast Cancer Res Treat 127(1):163-170

38. Armando E, Giuliano KV, Ballman, Linda, McCall et al (2017) Effect of Axillary Dissection vs No Axillary Dissection on 10-Year Overall Survival Among Women With Invasive Breast Cancer and Sentinel Node Metastasis The ACOSOG Z0011 (Alliance) Randomized Clinical Trial. JAMA 318(10):918-926

39. Jessica I, Goldberg ER, Riedel M, Morrow et al (2011) Morbidity of Sentinel Node Biopsy: Relationship Between Number of Excised Lymph Nodes and Patient Perceptions of Lymphedema. Ann Surg Oncol 18:2866-2872

40. Mitsuo Terada A, Yoshimura M, Sawaki et al (2020) Patient-reported outcomes and objective assessments with arm measurement and bioimpedance analysis for lymphedema among breast cancer survivors. Breast Cancer Res Treat 179(1):91-100 
41. Ballardini B, Santoro L, Sangalli C et al (2013) The indocyanine green method is equivalent to the $99 \mathrm{mTc}$-labeled radiotracer method for identifying the sentinel node in breast cancer: A concordance and validation study. Eur J Surg Oncol 39(12):1332-1336

42. Stephanie A, Valente Z, Al-Hilli, Diane M, Radford et al (2019) Near Infrared Fluorescent Lymph Node Mapping with Indocyanine Green in Breast Cancer Patients: A Prospective Trial. J Am Coll Surg 228(4):672-678

\section{Figures}

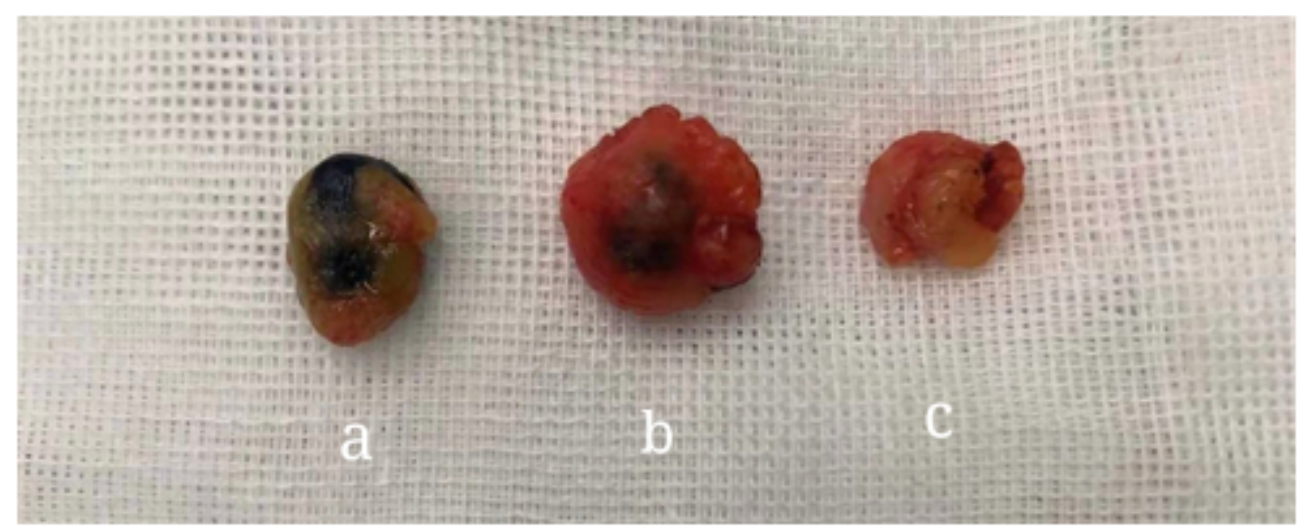

\section{(1) $\mathrm{MB}$}

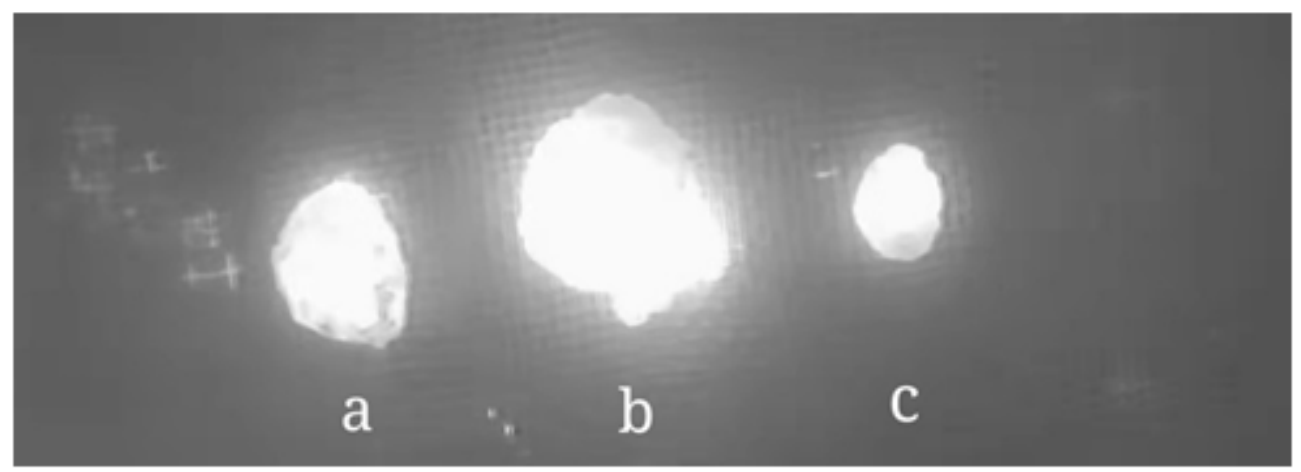

\section{(2) ICG}

\section{Figure 1}

Lymph node labeled by MB and/or ICG. Three sentinel lymph nodes were identified. Lymph node $a, b$ were labeled as $\mathrm{MB}(+) / \mathrm{ICG}(+)$, lymph node $\mathrm{c}$ was labeled as $\mathrm{MB}(-) / \mathrm{ICG}(+)$. 


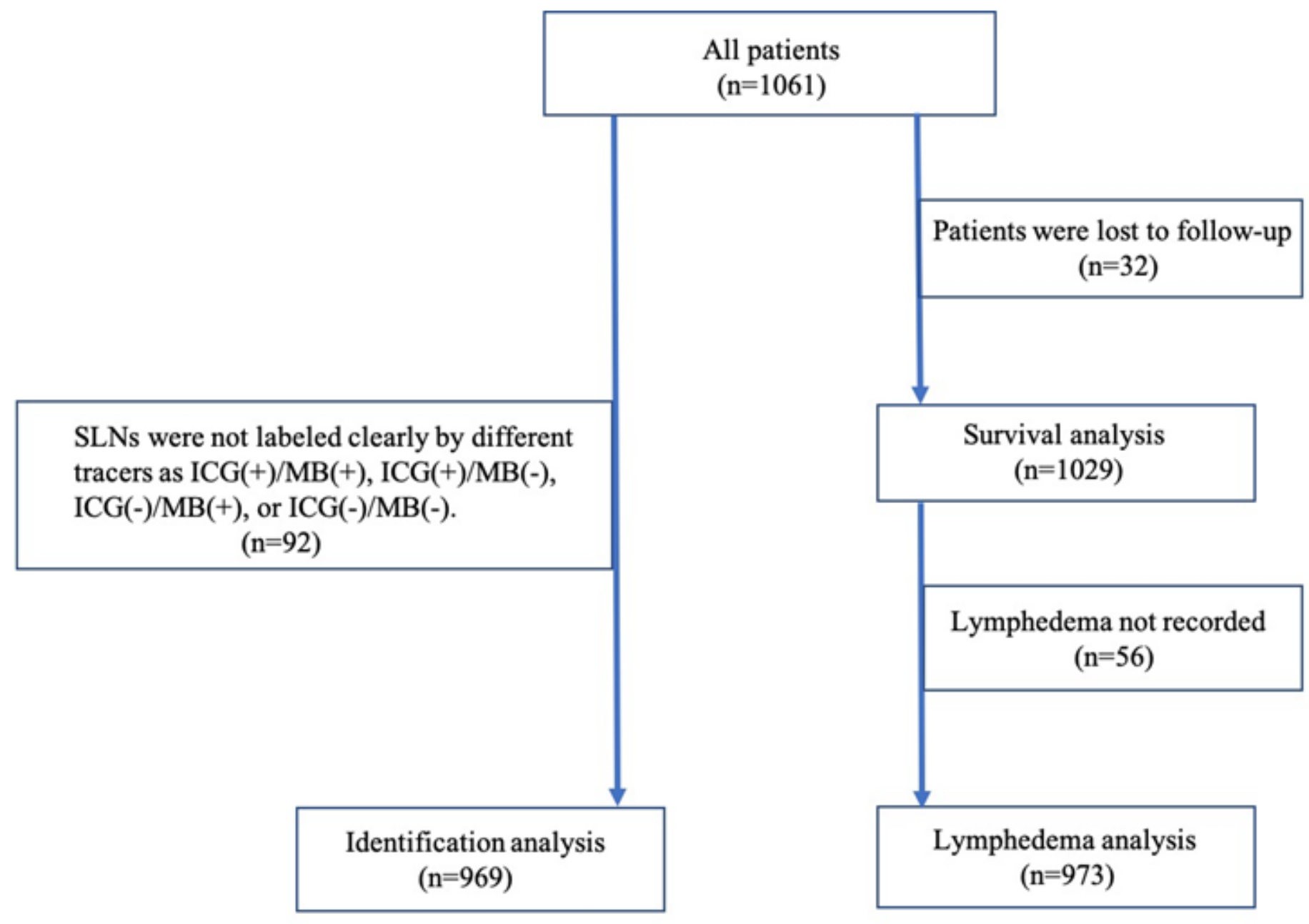

Figure 2

Study enrollment flow chart A total of 1061 patients were included, sentinel lymph nodes (SLNs) of 92 patients were not labeled clearly, but follow-up were performed. 32 patients were lost to follow-up, but SLNs of them were labeled clearly. Therefore, different analyses were performed in different populations. 


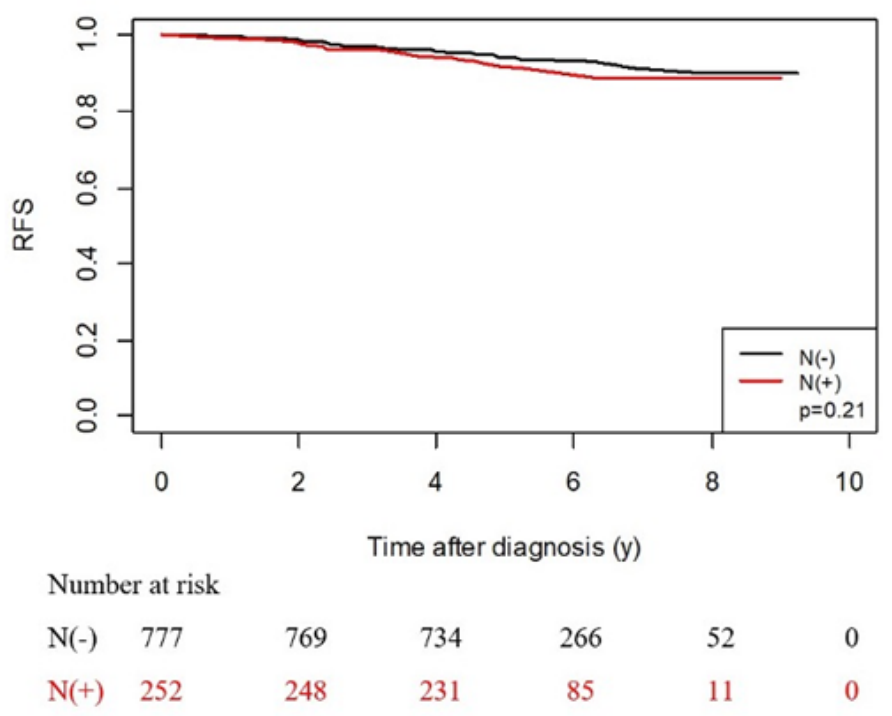

a Relapse Free Survival

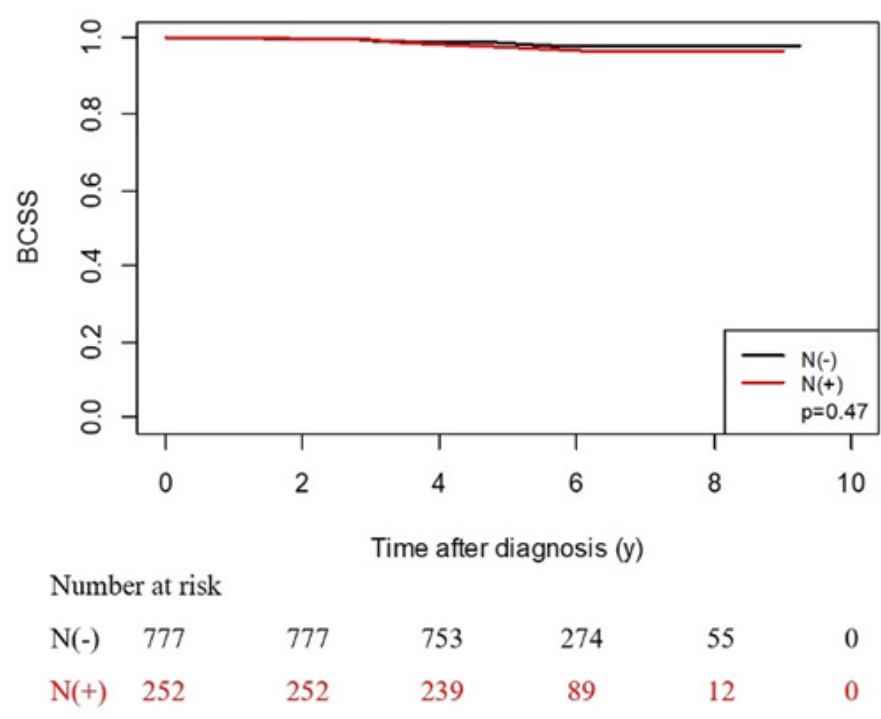

b Breast Cancer Specific Survival

\section{Figure 3}

Kaplan-Meier Estimates of Relapse Free Survival and Breast Cancer Specific Survival Panels a and b show relapse free survival (RFS) and breast cancer specific survival (BCSS), respectively. N(-) denotes lymph nodes negative and $\mathrm{N}(+)$ denotes lymph nodes positive. 